OPEN ACCESS

Edited by:

Peter Hamar,

Semmelweis University, Hungary

Reviewed by:

Francesco Pepe

University of Naples Federico II, Italy

Tiago Felismino,

AC Camargo Cancer Center, Brazi

${ }^{*}$ Correspondence:

Fang Wu

wufang4461@csu.edu.cn

Specialty section:

This article was submitted to

Cancer Immunity and Immunotherapy,

a section of the journal

Frontiers in Oncology

Received: 21 October 2021 Accepted: 24 January 2022

Published: 07 March 2022

Citation:

Hu C-H, Shi S, Dong W, Xiao L, Zang $H$ and Wu $F$ (2022) Hyperprogressive Disease After Immunotherapy:

A Case Report of Pulmonary Enteric Adenocarcinoma.

Front. Oncol. 12:799549. doi: 10.3389/fonc.2022.799549

\section{Hyperprogressive Disease After Immunotherapy: A Case Report of Pulmonary Enteric Adenocarcinoma}

\author{
Chun-Hong $\mathrm{Hu}^{1}$, Shenghao Shi ${ }^{1}$, Wen Dong ${ }^{2}$, Lizhi Xiao ${ }^{3}$, Hongjing Zang ${ }^{4}$ and Fang $\mathrm{Wu}^{1 \star}$ \\ 1 Department of Oncology, The Second Xiangya Hospital, Central South University, Changsha, China, ${ }^{2}$ Department of Oncology, \\ The Changde First People's Hospital, Changde, China, ${ }^{3}$ Department of Radiology, Second Xiangya Hospital, Central South \\ University, Changsha, China, ${ }^{4}$ Department of Pathology, Second Xiangya Hospital, Central South University, Changsha, China
}

Primary pulmonary enteric adenocarcinoma (PEAC) is a rare invasive adenocarcinoma clinically similar to metastatic colorectal adenocarcinoma (MCRC). Although many studies have addressed the differential diagnosis of PEAC, few have described the treatment of PEAC, especially using immunotherapy. This report describes a 61-year-old man who presented initially with pain in the ribs. Pathological analysis of biopsy samples shows malignant tumors of the right pleura, and next-generation sequencing of 26 genes showed a KRAS gene mutation. Positron emission tomography-computed tomography (PET-CT) found no evidence of gastrointestinal malignancy. Due to multiple metastases, the patient could not undergo radical surgery. The patient was treated with a combination chemotherapy regimen of paclitaxel plus carboplatin, along with sindilizumab immunotherapy, but, after one cycle of treatment, the tumor showed a hyperprogressive state. The patient is still being monitored regularly. These findings indicate that chemotherapy combined with immunotherapy may be ineffective in the treatment of primary PEAC with positive driver genes.

Keywords: pulmonary enteric adenocarcinoma, chemotherapy, immunity therapy, hyperprogressive disease, case report

\section{INTRODUCTION}

Pulmonary enteric adenocarcinoma (PEAC) is a very rare type of lung adenocarcinoma, first reported in 1991 (1). The 2015 World Health Organization (WHO) classification has defined PEAC as a primary lung adenocarcinoma containing more than $50 \%$ of intestinal differentiation components, with the tumor cells being positive for one or more immunohistochemical markers of gastrointestinal tumors. The pathogenesis of PEAC has not been fully determined, and there is currently no specific treatment plan. At present, the main treatment methods for PEAC are surgery and systemic chemotherapy. Although many case reports and studies have described the detection of different gene mutations in patients with PEAC, few have been administered targeted therapy (2).

The nonsynonymous tumor mutation burden (TMB) has been reported higher in patients with primary PEAC than in patients with ordinary lung adenocarcinoma, suggesting that patients with primary PEAC may be more likely to benefit from checkpoint blocking immunotherapy. Very few reports to date have described the effects of treatment with immunotherapy in patients with primary 
PEAC. The present report describes a patient with a rare primary lung and bowel adenocarcinoma who experienced tumor superprogression after chemotherapy combined with immunotherapy for primary PEAC.

\section{CASE DESCRIPTION}

A 61-year-old man visited the Second Xiangya Hospital of Central South University due to right chest pain for more than 4 months. In January 2021, he was seen at a local hospital; at that time, the patient reported having no obvious paroxysmal dull pain in the right chest or radiating pain between MS0-3. The patient had a dry cough, but no evidence of hemoptysis, hematemesis, chills, fever, or fatigue. Oral medication suggested by the local clinic was ineffective. At the beginning of March 2021, the patient visited a local hospital due to worsening pain on his right side, but there was no evidence of chills, fever, nausea, vomiting, dizziness, headache, abdominal pain, or diarrhea. The patient was admitted to the Department of Respiratory Medicine at a higher-level hospital on March 11, 2021, for further diagnosis and treatment. Physical examination at admission showed no obvious positive signs, and his ZPS score was 2 points. He was generally in good health and had no history of smoking or drinking. His parents died of unknown causes, although they had conditions similar to that of the patient. His family history included chronic diseases. His older sister died of "lung cancer," and his younger brother had been diagnosed with "tongue cancer." He reported no family history of genetic or infectious diseases. Computed tomography (CT) imaging of his lungs showed two lesions consistent with pneumonia and pleural effusion on his right side. Bronchoscopy showed multiple areas of mucosal irregularities in his visceral and parietal pleura, as well as multiple adhesive tapes between the visceral and parietal membranes. Examination of drained intracavitary fluid showed significant increases in tumor markers of pleural effusion, suggesting that this patient had malignant pleural effusion. Laparoscopy showed that the pleura occupied space, with the pathological diagnosis (Figure 1) being a malignant tumor in the right pleura. Immunohistochemical assays showed that the tumor was positive for CK7, CK19, and pd-11 (CPS 5); focally positive for CDX-2 (Figure 2) and villin; and negative for napsin $\mathrm{A}$ and CK20. These findings, along with a thoracoscopic biopsy of his right chest showing a malignant tumor, were consistent with a diagnosis of mucoepidermoid carcinoma. Further pathological examination resulted in a diagnosis of a poorly differentiated intestinal-type adenocarcinoma of the right pleura. Next-generation sequencing (NGS) of 26 genes associated with lung cancer showed that this tumor was positive for a KRAS mutation (Supplementary Materials). Due to the possibility of metastasis, the patient underwent positron emission tomography (PET)-CT examination in our hospital. The results showed that the right pleura were extensively thickened and glucose metabolism had increased, findings consistent with membranous malignant tumors suggestive of metastases. In addition, the lymph nodes in the right hilum and mediastinum (zone 4) were slightly enlarged, with increased glucose metabolism, suggesting lymph node metastases. Sternal bodies and multiple thoracic and lumbar spine attachments were observed, with bones and right bone wings showing increased glucose metabolism and bone destruction, suggesting bone metastases. Gastrointestinal lesions, however, were not observed. These pathological and immunohistochemical findings excluded lung metastases of intestinal adenocarcinoma, and the patient was finally diagnosed with pulmonary intestinal adenocarcinoma.

As the patient had multiple metastases at presentation, radical surgery was no longer possible. Before the diagnosis, the patient received symptomatic treatment to relieve pain. After the diagnosis, the patient and his family were informed of the

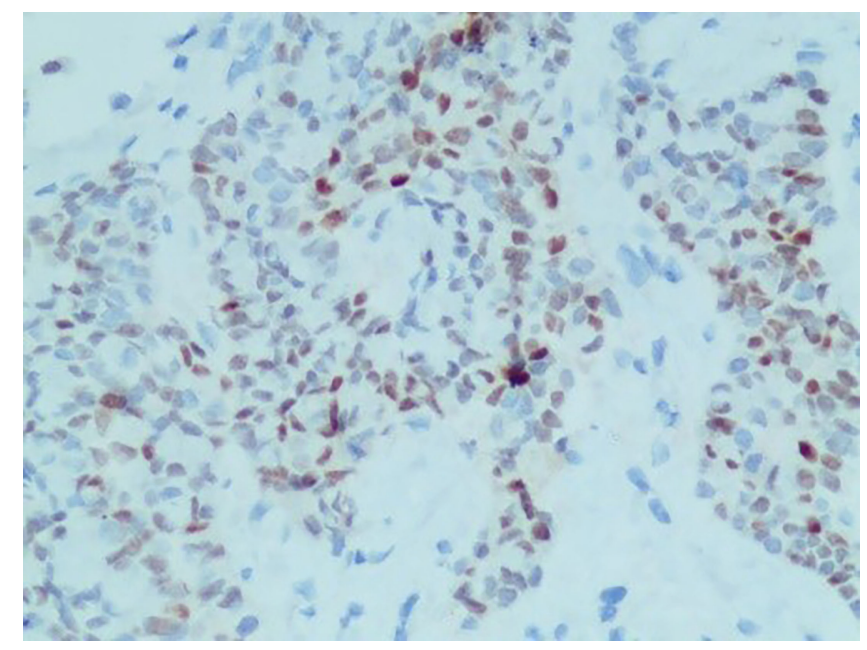

FIGURE 1 | IHC: CDX-2 (+). 


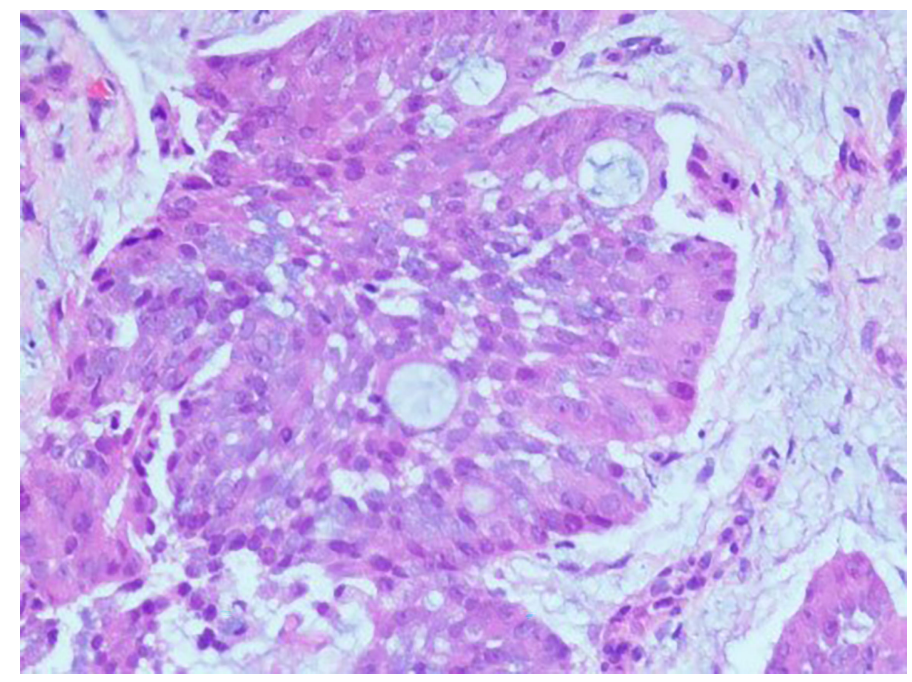

FIGURE 2 | Pathology imagine.

necessity of for antitumor therapy and its possible side effects, including myelosuppression, immune-related pneumonia, immune-related myocarditis, and liver damage. The patient and family members expressed their understanding and agreed to systemic chemotherapy plus sindilizumab immunotherapy. After one cycle of treatment, the patient reported poor pain control and a weight loss of about $3 \mathrm{~kg}$. Repeat chest and abdominal CT showed significant thickening of the right pleura (Figures 3, 4), indicating that the disease had progressed rapidly and that patient prognosis was extremely poor. Although a second-line treatment regimen was selected, consisting of paclitaxel combined with anlotinib, the patient and family members refused further treatment, as the patient was less likely to benefit from this regimen than from the first-line regimen. At this writing, the patient remains alive but in generally poor condition and continues to receive nutritional support at a local hospital.

\section{DISCUSSION}

In 2011, the International Association for the Study of Lung Cancer, the American Thoracic Society, and the European Respiratory Society jointly issued guidelines for the

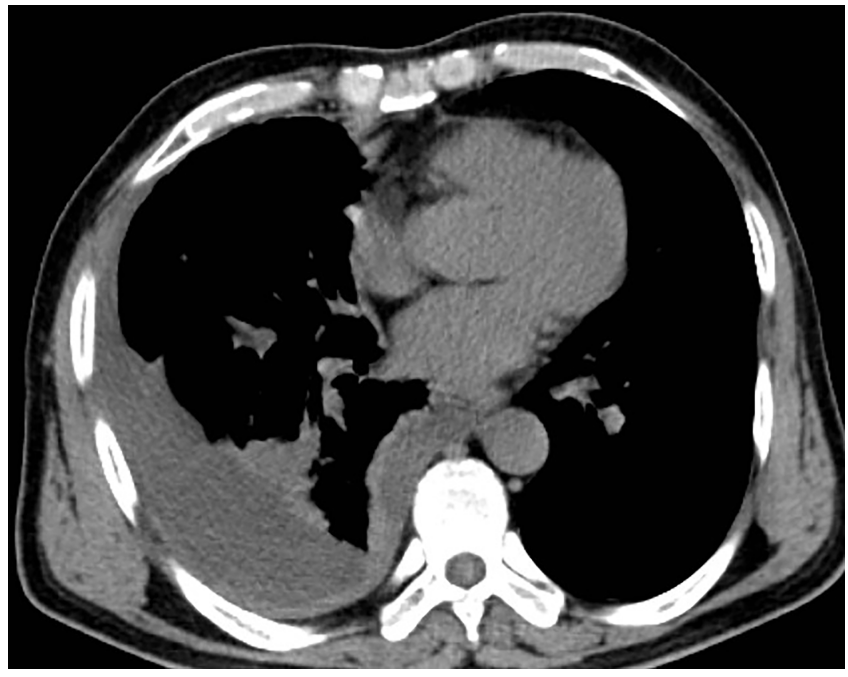

FIGURE 3 | CT before immunotherapy. 


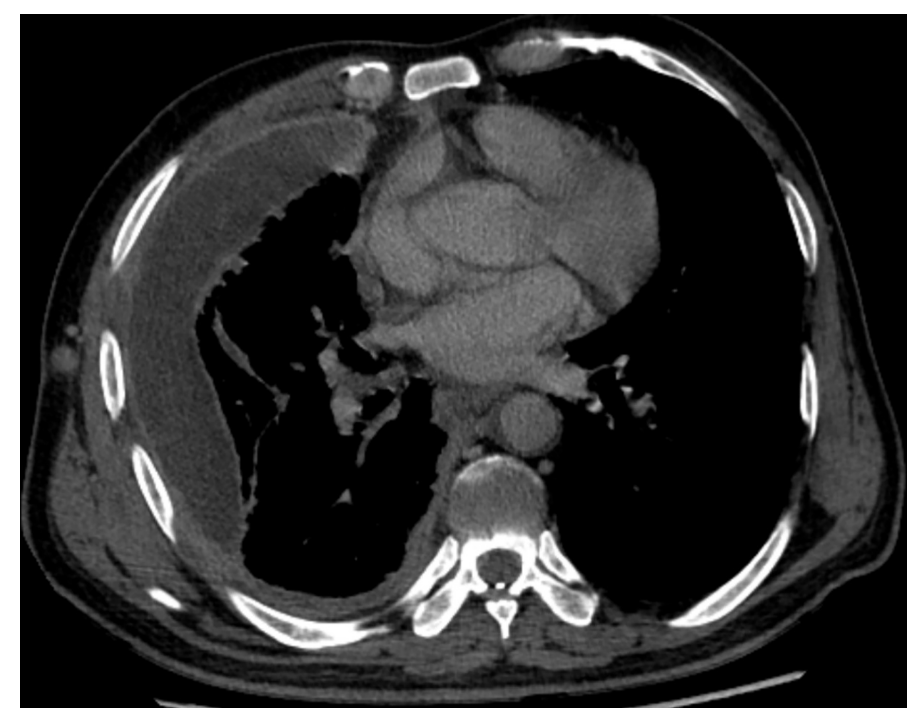

FIGURE 4 | CT after immunotherapy.

multidisciplinary classification of lung adenocarcinomas, enabling better treatment planning treatment and guiding prognosis (3). The treatment strategy for lung-intestinal adenocarcinoma, a rare type of lung adenocarcinoma, is similar to that for nonsmall cell lung cancer and can include various combinations of surgery, chemotherapy, radiotherapy, and targeted therapy (4). Patients with late stage tumors are ineligible for surgery can be treated successfully with four cycles of pemetrexed and carboplatin chemotherapy (5) or paclitaxel combined with cisplatin (6). Most lung and bowel adenocarcinomas are negative for EGFR mutations but positive for KRAS mutations (7), suggesting that tyrosine kinase inhibitors would be ineffective in these patients.

The 2021 Chinese Society of Clinical Oncology (CSCO) guidelines for the diagnosis and treatment of primary lung cancer have recommended immunosuppressants such as pembrolizumab as first-line treatment for patients with stage IV nonsquamous cell nonsmall cell lung cancer (NSCLC) without driver genes. PD-1 is highly expressed in PEACs, and nonsynonymous TMB is significantly higher in patients with primary PEAC than in patients with ordinary lung adenocarcinomas, suggesting that patients with PEAC may benefit from treatment with immune checkpoint inhibitors (8).

Although immunotherapy should have relatively good therapeutic potential in patients with lung-intestinal adenocarcinoma (9), the combination of immunotherapy and chemotherapy in the present patient resulted in tumor hyperprogression. Although hyperprogressive disease (HPD) after immunotherapy has not been defined precisely, it is thought to include (1) a ratio of tumor growth kinetic (TGK) variables during immunotherapy to pretreatment TGK $>2$ (10); (2) a tumor growth rate (TGR) $\geq 2$-fold higher after than before immunotherapy (11); (3) the combination of a treatment failure time (TTF) $<2$ months, a $>50 \%$ increase in tumor burden, and a TGR $>2$ after chemotherapy (12); (4) the combination of a
TTF $<2$ months, a $\geq 50 \%$ increase in total diameter of the target lesions, and the appearance of at least two new lesions in the affected organs, a spread to new organs or worsened clinical manifestations, defined as a performance status (PS) $\geq 2$ (13); or (5) progressive disease (PD) during the first 8 weeks of treatment, including a $\geq 40 \%$ increase in the total diameter of the target lesions, or the appearance of at least two new lesions in different organs (14). Based on all of these standards, the present patient developed HPD after only one cycle of chemotherapy combined with immunotherapy. A meta-analysis of 1,389 NSCLC patients included in six studies found that the incidence of HPD ranged from $8.02 \%$ to $30.43 \%$, with overall survival rate being lower in patients with than without HPD. Risk factors for HPD included an Eastern Cooperative Oncology Group PS >1, a Royal Marsden Hospital score $\geq 2$, a serum lactate dehydrogenase concentration above the upper limit of normal, more than two metastatic sites, and liver metastases (15). Other risk factors for HPD included age $>65$ years, gender, smoking history, neutrophil-to-lymphocyte ratio (NLR), PD1/PD-L1, PDL1 status, monotherapy/combination therapy, and previous line of treatment. In contrast, HPD was not significantly associated with tumor number, histopathological type of NSCLC, EGFR mutation, KRAS mutation, or ALK rearrangement.

Hyperprogression in response to immunotherapy may have been due to driver gene mutations. For example, systemic chemotherapy treatment of a patient with advanced lungintestinal adenocarcinoma and a new KRAS Q22K mutation resulted in rapid disease progression, with the patient dying within a short time (16). Although KRAS dependence may have been the main genetic factor responsible for poor patient prognosis, the small number of patients with this condition prevents definitive determination of the relevant mechanism.

In summary, a patient with a primary PEAC and a KRAS mutation experienced super-progression after being treated with 
immunotherapy combined with chemotherapy. Few reports to date have described the association between immunotherapy and outcomes in patients with lung-intestinal adenocarcinoma, especially in patients with treatment-associated HPD. The findings observed in this patient, including diagnosis, treatment, and the association between outcomes and driver genes may lead to studies on the treatment and prognosis of patients with lung and bowel adenocarcinoma.

\section{DATA AVAILABILITY STATEMENT}

The original contributions presented in the study are included in the article/Supplementary Material. Further inquiries can be directed to the corresponding author.

\section{ETHICS STATEMENT}

Written informed consent was obtained from the individual(s) for the publication of any potentially identifiable images or data included in this article.

\section{REFERENCES}

1. Tsao MS, Fraser RS. Primary Pulmonary Adenocarcinoma With Enteric Differentiation. Cancer (1991) 68:1754-7. doi: 10.1002/1097-0142(19911015) 68:8<1754::aid-cncr2820680818>3.0.co;2-e

2. Li H, Cao W. Pulmonary Enteric Adenocarcinoma: A Literature Review. J Thorac Dis (2020) 12:3217-26. doi: 10.21037/jtd-19-4171

3. Travis WD, Brambilla E, Noguchi M, Nicholson AG, Geisinger K, Yatabe Y, et al. American Thoracic Society. International Association for the Study of Lung Cancer/American Thoracic Society/European Respiratory Society: International Multidisciplinary Classification of Lung Adenocarcinoma: Executive Summary. Proc Am Thorac Soc (2011) 8:381-5. doi: 10.1513/pats.201107-042ST

4. Gong J, Fan Y, Lu H. Pulmonary Enteric Adenocarcinoma. Transl Oncol (2021) 14:101123. doi: 10.1016/j.tranon.2021.101123

5. Li HC, Schmidt L, Greenson JK, Chang AC, Myers JL. Primary Pulmonary Adenocarcinoma With Intestinal Differentiation Mimicking Metastatic Colorectal Carcinoma: Case Report and Review of Literature. Am J Clin Pathol (2009) 131:129-33. doi: 10.1309/AJCPB04XWICTFERL

6. Lin LI, Xu CW, Zhang BO, Liu RR, Ge FJ, Zhao CH, et al. Clinicopathological Observation of Primary Lung Enteric Adenocarcinoma and Its Response to Chemotherapy: A Case Report and Review of the Literature. Exp Ther Med (2016) 11:201-7. doi: 10.3892/etm.2015.2864

7. Gu L, Wang XZ, Wen W, Lin J, Chen XF, Lai GX, et al. Clinical Analysis of 23 Patients Pathologically Diagnosed With Primary and Secondary Pulmonary Enteric Adenocarcinoma. Chin Med J (Engl) (2019) 132):1368-9. doi: 10.1097/CM9.0000000000000266

8. Chen M, Liu P, Yan F, Xu S, Jiang Q, Pan J, et al. Distinctive Features of Immunostaining and Mutational Load in Primary Pulmonary Enteric Adenocarcinoma: Implications for Differential Diagnosis and Immunotherapy. J Transl Med (2018) 16:81. doi: 10.1186/s12967-018-1449-Z

9. Jurmeister P, Vollbrecht C, Behnke A, Frost N, Arnold A, Treue D, et al. Next Generation Sequencing of Lung Adenocarcinoma Subtypes With Intestinal Differentiation Reveals Distinct Molecular Signatures Associated With Histomorphology and Therapeutic Options. Lung Cancer (2019) 138:43-51. doi: 10.1016/j.lungcan.2019.10.005

10. Saada-Bouzid E, Defaucheux C, Karabajakian A, Coloma VP, Servois V, Paoletti X, et al. Hyperprogression During Anti-PD-1/PD-L1 Therapy in Patients With Recurrent and/or Metastatic Head and Neck Squamous Cell Carcinoma. Ann Oncol (2017) 28:1605-11. doi: 10.1093/annonc/mdx178

\section{AUTHOR CONTRIBUTIONS}

FW: were responsible for study conception and design and acquiring financial support. WD: provided patient information. SS: design, interpretation, and write-up of final article. All authors contributed to the article and approved the submitted version.

\section{ACKNOWLEDGMENTS}

The authors thank Lizhi Xiao of the Department of Radiology, the Second Xiangya Hospital, Central South University, for imaging analysis and advice and Hong Jing of the Department of Pathology, the Second Xiangya Hospital, Central South University, for histologic analysis and advice and for proofreading the final version of this article.

\section{SUPPLEMENTARY MATERIAL}

The Supplementary Material for this article can be found online at: https://www.frontiersin.org/articles/10.3389/fonc.2022. 799549/full\#supplementary-material

11. Champiat S, Ferrara R, Massard C, Besse B, Marabelle A, Soria JC, et al. Hyperprogressive Disease: Recognizing a Novel Pattern to Improve Patient Management. Nat Rev Clin Oncol (2018) 15:748-62. doi: 10.1038/s41571-018-0111-2

12. Kato S, Goodman A, Walavalkar V, Barkauskas DA, Sharabi A, Kurzrock R. Hyperprogressors After Immunotherapy: Analysis of Genomic Alterations Associated With Accelerated Growth Rate. Clin Cancer Res (2017) 23:4242 50. doi: 10.1158/1078-0432.CCR-16-3133

13. Lo Russo G, Moro M, Sommariva M, Cancila V, Boeri M, Centonze G, et al. Antibody-Fc/FcR Interaction on Macrophages as a Mechanism for Hyperprogressive Disease in Non-Small Cell Lung Cancer Subsequent to PD-1/PD-L1 Blockade. Clin Cancer Res (2019) 25:989-99. doi: 10.1158/1078-0432.CCR-18-1390

14. Matos I, Martin-Liberal J, Garcia-Ruiz A, Hierro C, Ochoa de Olza M, Viaplana C, et al. Capturing Hyperprogressive Disease With ImmuneCheckpoint Inhibitors Using RECIST 1.1 Criteria. Clin Cancer Res (2020) 26:1846-55. doi: 10.1158/1078-0432.CCR-19-2226

15. Chen Y, Hu J, Bu F, Zhang H, Fei K, Zhang P. Clinical Characteristics of Hyperprogressive Disease in NSCLC After Treatment With Immune Checkpoint Inhibitor: A Systematic Review and Meta-Analysis. BMC Cancer (2020) 20:707. doi: 10.1186/s12885-020-07206-4

16. Metro G, Valtorta E, Siggillino A, Lauricella C, Cenci M, Ludovini V, et al. EntericType Adenocarcinoma of the Lung Harbouring a Novel KRAS Q22K Mutation With Concomitant KRAS Polysomy: A Case Report. Ecancermedicalscience (2015) 9:559. doi: 10.3332/ecancer.2015.559

Conflict of Interest: The authors declare that the research was conducted in the absence of any commercial or financial relationships that could be construed as a potential conflict of interest.

Publisher's Note: All claims expressed in this article are solely those of the authors and do not necessarily represent those of their affiliated organizations, or those of the publisher, the editors and the reviewers. Any product that may be evaluated in this article, or claim that may be made by its manufacturer, is not guaranteed or endorsed by the publisher.

Copyright (c) $2022 \mathrm{Hu}$, Shi, Dong, Xiao, Zang and Wu. This is an open-access article distributed under the terms of the Creative Commons Attribution License (CC BY). The use, distribution or reproduction in other forums is permitted, provided the original author(s) and the copyright owner(s) are credited and that the original publication in this journal is cited, in accordance with accepted academic practice. No use, distribution or reproduction is permitted which does not comply with these terms. 